\title{
Upper body strength endurance evaluation: A comparison between the handgrip strength and three body weight tests
}

Ewan Thomas*, Vincenzo Gennaro, Giuseppe Battaglia, Marianna Bellafiore, Angelo Iovane, Antonio Palma and Antonino Bianco Sport and Exercise Research Unit, Department of Psychological, Pedagogical and Educational Sciences, University of Palermo, Palermo, Italy

Received 29 July 2020

Accepted 9 October 2020

\section{Abstract.}

BACKGROUND: The hand-grip strength test has been widely adopted to evaluate upper limb strength. Other field based tests as push-ups and pull-ups are commonly used for the same purpose. It is however unclear if these may be used interchangeably for upper body strength evaluation.

OBJECTIVE: The purpose of this investigation was to evaluate strength endurance of the upper body and understand which test could be the most appropriate for upper body evaluation.

METHODS: Thirty-eight healthy young male participants were tested with three tests comprised of: 1) push-ups (PS), 2) pull-ups (PL) and 3) parallel dips (PD) performed to exhaustion. Grip strength (GS), total number of repetitions, time-to-complete the test, repetition cadence and rate of perceived exertion (RPE) were also retrieved for investigation.

RESULTS: Repetitions, time-to-complete the test and repetition cadence significantly differed across the three tests $(p<0.001)$. No difference in the RPE was present. No correlation was present between GS and the other tests. No correlation was present between RPE and performance values and time-to-complete the tests. BMI was positively correlated to RPE in all tests. All tests strongly correlate to each other (PS vs. PL $r=0.55$; PS vs. PD $r=0.64$; PL vs. PD $r=0.70$ ) and to time-to-complete the test (PS $r=0.79$; PL $r=0.69$; PD $r=0.66$ ). Only the results of the PD correlate to their respective repetition cadence $(r=0.66)$. CONCLUSIONS: GS is not suitable to evaluate strength endurance. PS, PL and PD are all suitable to evaluate strength endurance. However, PD may be preferred to evaluate the upper body, if velocity also needs to be taken into account.

Keywords: Strength endurance, upper body, strength, push-ups, pull-ups, parallel dips

\section{Introduction}

It is well established that strength is one of the most important health related aspects in humans [1,2]. Indeed extensive literature has been carried out over the years

${ }^{*}$ Corresponding author: Ewan Thomas, Sport and Exercise Research Unit, Department of Psychological, Pedagogical and Educational Sciences, University of Palermo, Via Giovanni Pascoli 6, Palermo, 90144, Italy. Tel.: +39 3208899934; E-mail: ewan.thomas @unipa.it. in order to understand maximal strength and define neuromuscular function $[3,4]$. Nonetheless, an equally important parameter of neuromuscular function is strength endurance, which has received less attention in the literature [5].

Strength endurance is defined as the ability of muscles to repeatedly exert muscular force for an extended period [6]. This aspect of strength was also identified by a review from de la Motte et al. [7] as an independent risk factor for musculoskeletal injury. The authors evaluated the association between strength endurance and 
musculoskeletal injuries, indicating that men with lower strength endurance had an overall increased risk of injury. Strength endurance therefore represents a measure of functional capacity which is specific for each muscle group [5]. This concept also implies the necessity for different tests to be adopted to evaluate different muscle groups.

A reliable technique to evaluate strength endurance consists in assessing maximal strength through a dynamometer or a 1RM test and based on a percentage of this value (i.e. 5 or 10\%), to evaluate time to failure of a sustained isometric contraction [8-10]. This technique however requires the use of a dynamometer or specific equipment in a laboratory setting environment. Other common assessment methods employ field based tests, as push-ups, squats and sit-ups [11] which are those most frequently adopted, for upper body, lower body and core muscle evaluation, respectively. These strength endurance tests are performed either against time, by evaluating the maximum number of repetitions executed within 60 seconds [12,13], or by determining the maximum number of repetitions regardless of time, until exhaustion $[14,15]$.

In the context of upper body strength endurance testing, two common tests are the push-up and pullup tests, for pushing and pulling strength, respectively [11,16-19]. Another common exercise proposed to improve upper body strength, which can be also employed as a mean of evaluation, is the parallel bar dip [20,21]. However, only Collins et al. [22] and Paoli et al. [23] have considered the use of a parallel bar dip test, performed to exhaustion, to evaluate upper body strength endurance. Interestingly, notwithstanding different populations were analysed by the two studies, very similar results were obtained regarding the test results.

Therefore, the aim of this investigation will be to assess strength endurance of the upper body in healthy young males and to identify which test could be the most suitable for an overall general upper body strength evaluation.

\section{Materials and methods \\ 2.1. Subjects}

The sample was composed of 38 young male healthy participants (age $23.9 \pm 6.7$ years; weight $70.7 \pm$ $11.9 \mathrm{~kg}$; height $172.8 \pm 6.9 \mathrm{~cm}$ ). The participants were all recruited within fitness centres and were eligible to participate if they were free of injuries or illnesses.
The participants were excluded if they were unable to perform the required tasks and if their training experience was less than three months in resistance training or body weight training. Each participant was informed about the risks and benefits of participating in this study prior to providing informed written consent. This was mandatory to participate in the study.

The principles of the Italian data protection (196/ 2003) were guaranteed. The study was undertaken in accordance with the guidelines of the Helsinki Declaration (Hong Kong revision, September 1989) and the European Union recommendations for Good Clinical Practice (document 111/3976/88, July 1990).

\subsection{Procedure}

Data collection was carried out by two investigators in the setting of a fitness center. The first step consisted of measuring anthropometric parameters of each participant. Subsequently, each participant was asked to perform the handgrip strength test (GS) three times with the right and left hand. A two minute rest was provided between each GS trial. At the end of the GS assessment, further 5-minutes rest were given before the subsequent tests were administered.

Three tests were administered in a random order. They were push-ups (PU), pull-ups (PL) and parallel bar dips tests (PD), all performed to exhaustion. Each test was performed on a separate day and all tests were performed on non-consecutive days in order to allow a full recovery of the participants. Each test was performed once, starting at the "go" of an investigator and ending when either the participant was not able to perform any more repetitions or when the repetitions were non performed as described in the following section, for more than two consecutive repetitions.

The other investigator at the "go" started recording the time required to complete the task with a stopwatch, to the nearest hundredth of a second, which was stopped at the end of the test. No restrains on the execution speed were made in order to allow subject's preferred cadence. Once the participant ended the required task, the rate of perceived exertion was assessed. This procedure was repeated for each test. At the end of data collection, repetition cadence was calculated for each test and participant.

\subsection{Measures}

\subsubsection{Handgrip strength test}

Hand-grip strength was measured through a digital dynamometer (KERN MAP 80K1, KERN\&Sohn 
$\mathrm{GmbH}$, Barlinger, Germany). Each participant performed three trials with both hands with a two-minute rest between each trial. The participants were instructed to hold the dynamometer in each hand, with the arm fully extended and were instructed to hold the dynamometer without touching the body. The display of the dynamometer was aligned to the face of the examiner. The participants were standing during the entire test with the arm straight down at the side, the elbow in full extension and the forearm and the wrist in neutral position. The highest of the three trials was recorded for statistical analysis.

\subsubsection{Push-up test}

The push-up test was performed on a flat, stable surface, with the hands placed slightly wider than shoulderwidth apart. The fingers were pointing forward and the body parallel to the ground. For the repetition to be recorded, the correct depth needed to be met. This was reached when each elbow formed an angle of at least $90^{\circ}$ during the eccentric phase of the movement. The test ended when the participants were no longer able to perform additional repetitions.

\subsubsection{Pull-up test}

The pull-up test was performed with each participant grasping an overhead bar with a pronated grip. For each pull-up the participants had to start from a motionless hanging position from a $2.15 \mathrm{~m}$ high bar with the upper limbs fully extended. The participants had to pull up their body until at least their chin passed above the bar. The participants weren't allowed to swing or use their legs in order to provide help during the execution of the test. The test ended when the participants were no longer able to perform additional repetitions or if they used their legs for help during the execution of the test.

\subsubsection{Parallel bar dip test}

The parallel bar dip test was performed with each participant on a set of parallel bars, $55 \mathrm{~cm}$ wide and $140 \mathrm{~cm}$ high. The participants started the test while with the arms fully extended, grabbing with each hand a parallel bar. For the repetition to be recorded, the correct depth needed to be met, and this was reached when each elbow formed an angle of at least $90^{\circ}$ during the eccentric phase of the movement. During the concentric phase the participants were not allowed to use their legs to provide help during the execution of the test. The test ended when the participants were no longer able to perform additional repetitions or when they used their legs for help.
Table 1

Descriptive characteristics of the sample and main measures

\begin{tabular}{lccc}
\hline \multicolumn{1}{c}{ Variables } & & \\
\hline Age (years) & $23.95 \pm 6.71$ & & \\
Weight (kg) & $70.74 \pm 11.09$ & & \\
Height (cm) & $172.76 \pm 6.96$ & & \\
GS R (kg) & $47.97 \pm 8.86$ & & Parallel dips \\
GS L (kg) & $45.12 \pm 8.47$ & & Pull-ups \\
\hline \multicolumn{5}{c}{ Push-ups } & & \\
\hline Repetitions & $52.29 \pm 14.35$ & $14.45 \pm 5.27$ & $27.11 \pm 11.18$ \\
Time (s) & $68.68 \pm 24.68$ & $43.04 \pm 16.78$ & $40.57 \pm 12.21$ \\
Cadence (reps/s) & $0.79 \pm 0.16$ & $0.35 \pm 0.10$ & $0.67 \pm 0.20$ \\
RPE & $16.45 \pm 2.23$ & $15.79 \pm 2.12$ & $16.74 \pm 1.67$
\end{tabular}

$\mathrm{R}=$ Right; $\mathrm{L}=$ Left; All data are presented as means \pm std.dv.

\subsubsection{Borg rate of perceived exertion scale}

Standardized written instructions were provided prior to each test in order to understand the BORG RPE scale. At the end of each test the participants had to rate the exertion of the test, using the BORG RPE scale ranging between 6 and 20 [24]. The results were recorded in an excel sheet.

\subsection{Statistical analysis}

Means and standard deviations were calculated from the current data. BMI was calculated from height and weight, and repetition cadence was calculated by dividing the number of total repetitions by the time required to complete the test. Data was then tested for normality using the Shapiro-Wilks test. All data ware normally distributed except for the data regarding the RPE. Differences between test results were calculated using a two-way ANOVA for parametric assessment and the Friedman test for non-parametric assessment. Pearson correlation coefficients and Spearman's rank correlation coefficients were also performed when appropriate. Linear regression models were subsequently created in order to verify which test had the greatest shared variance with the other tests. Significance was set at $\alpha 0.05$ for all analysis.

\section{Results}

Descriptive characteristics of the sample are presented in Table 1.

The number of repetitions performed was $52.29 \pm$ 14.35 for the PS, $14.45 \pm 5.27$ for the PL and 27.11 \pm 11.18 for the PD test (Fig. 1). A significant difference is present between the performance results of the three tests $(p<0.001)$. Also, time to complete the test (Fig. 2) and repetition cadence (Fig. 3) showed signifi- 
4 E. Thomas et al. / Upper body strength endurance evaluation

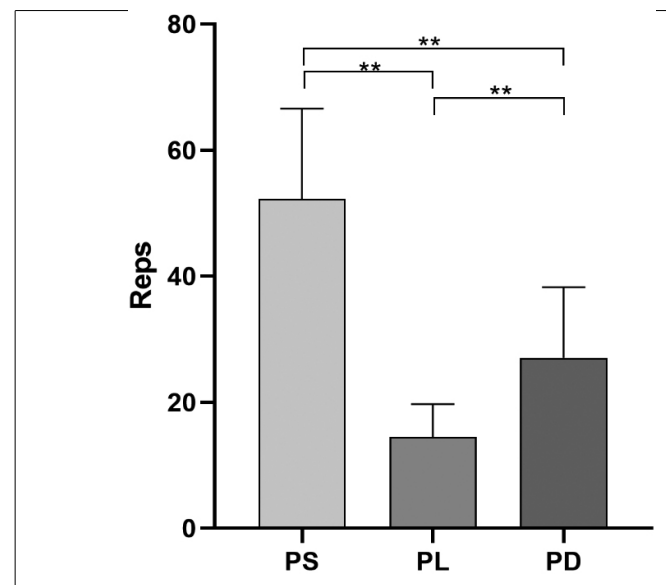

Fig. 1. Results of the performance of the three tests. PS = push-ups; $\mathrm{PL}=$ pull-ups; $\mathrm{PD}=$ parallel dips. ${ }^{* *} p<0.01$.

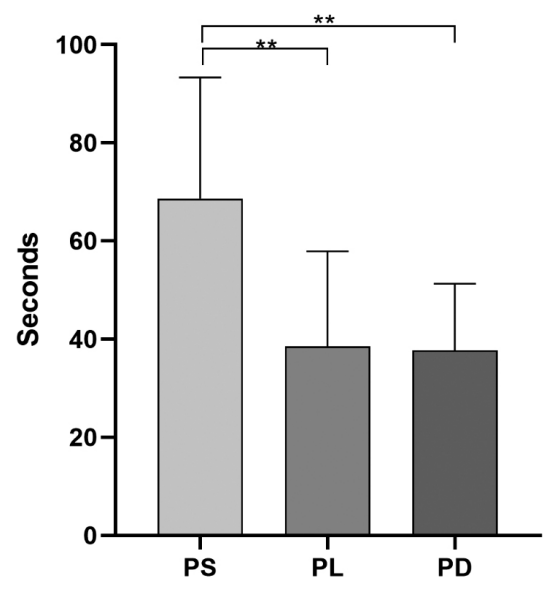

Fig. 2. Time required to complete the tests. PS = push-ups; PL = pull-ups; PD $=$ parallel dips. ${ }^{* *} p<0.01$.

cant differences between the tests $(p<0.001$ and $p<$ 0.001 , respectively). No difference however was seen for RPE values at the end of each test (Fig. 4).

None of the analysed tests significantly correlated to the GS for either hand, nor to BMI. However, BMI was significantly correlated to the RPE of each test (PS $r=0.62$; PL $r=0.64$ and PD $r=0.90)$. No correlation was found regarding the performance measures and RPE, nor between RPE and time to complete the tests $(r=0.12$ for PS, $r=-0.08$ for PL and $r=-0.32$ for PD), indicating that time was not the primary variable responsible for perceived exertion. While the performance measures correlated highly to the time required to complete the tests (Table 2), indicating that those who were able to sustain exercise for longer time performed more repetitions.

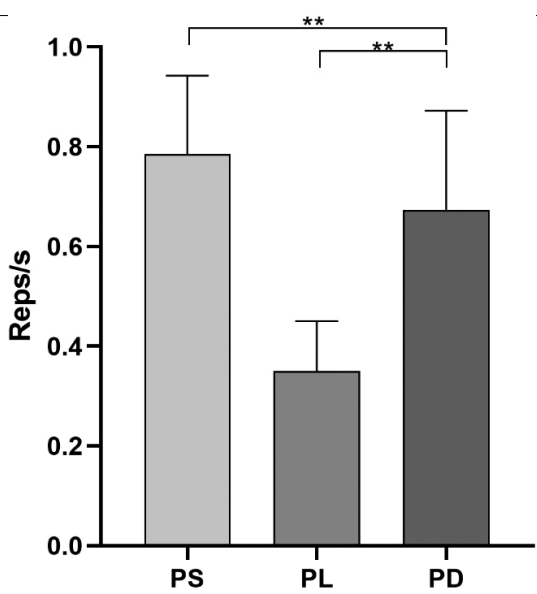

Fig. 3. Cadence, calculated by dividing the performance outcomes by time to complete the tests. PS = push-ups; PL = pull-ups; PD = parallel dips. ${ }^{* *} p<0.01$.

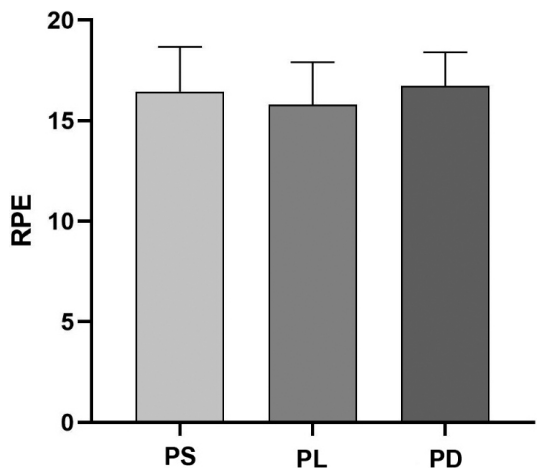

Fig. 4. Rate of perceived exertion of each test. PS = push-ups; PL = pull-ups; $\mathrm{PD}=$ parallel dips.

Table 2

Correlation coefficients of the analysed variables

\begin{tabular}{lccc}
\hline Variables & Push-ups & Pull-ups & Parallel dips \\
\hline GS R & -0.00 & 0.09 & 0.06 \\
GS L & -0.05 & -0.02 & -0.01 \\
Time & $0.79^{*}$ & $0.69^{*}$ & $0.66^{*}$ \\
Cadence & 0.19 & 0.35 & $0.66^{*}$ \\
RPE & -0.13 & -0.08 & -0.35 \\
Push-ups & 1 & $0.55^{*}$ & $0.64^{*}$ \\
Pull-ups & $0.55^{*}$ & 1 & $0.70^{*}$ \\
Dips & $0.64^{*}$ & $0.70^{*}$ & 1 \\
\hline
\end{tabular}

$\mathrm{R}=\mathrm{Right} ; \mathrm{L}=\mathrm{Left} ;{ }^{*}$ significant correlations.

Repetition cadence was not correlated to the performance measures of the PS and PL tests while a coefficient of $r=0.66$ was present for the PD, indicating a relation between the test results and velocity in this exercise.

Each test significantly correlated to each other (Table 2). A regression model was created in order to verify 
which test had the greatest shared variance. The PD had a significant $R^{2}=0.51$ with the PL and a significant $R^{2}=0.44$ with PS. Notwithstanding the shared variance between PS and PL was significant, a lower value was retrieved $\left(R^{2}=0.35\right)$.

\section{Discussion}

This study aimed to understand which exercise may be the most suitable in order to evaluate strength endurance of the upper body and the results of this study confirm that all three exercises may be adopted.

All exercises well correlate to each other and have a significant amount of shared variance.

Despite the challenge to precisely identify one exercise, it is interesting to note that the PD not only possess the highest partial correlations, but also the greatest shared variance with the other evaluated tests. Different elements need to be taken into account to understand these results. First, it is important to note that the PD is an exercise involving the upper body used to increase pushing strength, in which the main muscle groups engaged are the pectoralis major, the anterior deltoid and the triceps [25]. These muscle groups are those mainly engaged during the execution of the push-ups [26] and in part also during the pull-ups [27]. Furthermore, all exercises share common muscle groups which act as stabilizers during movement (i.e. rectus abdominis, erector spinae and serratus anterior) $[28,29]$. Second, the PD is executed on a frontal plane which is the same working plane used during the pull-ups, notwithstanding this latter is generally adopted for pulling strength [30]. Therefore, it was expected that a relation between the exercises was present.

In a previous study [14] we aimed to identify predicative variables for upper body strength endurance. The results indicated that velocity of a single repetition was the key variable identified in order to estimate the total number of performed repetitions during a pullup test. In the present investigation we did not assess velocity of single repetitions, however we estimated repetition cadence in order to identify further variables possibly related to velocity which don't need specific equipment to be calculated. The PD was the only exercise which manifested a positive and significant correlation to repetition cadence, highlighting a relation with velocity. Such strict relation between execution speed and performance output, was also evaluated by different authors. Zalleg et al. [31] identified through a principal component analysis that explosive push-ups were good estimators of upper body power while Sreckovic et al. [32] found evidence of a linear force-velocity relation regarding mechanical properties of arm muscles. All factors indicating that during muscular evaluation, velocity is an important component that should be further considered.

Another test included in our investigation was the GS, a gold standard in strength evaluation of the upper limbs, which is associated to several health related outcomes [33]. A study by Wind et al. [34] indicates grip strength may be used as a predictor of general muscle strength in different populations. However, in their investigation the authors only considered isometric strength without taking into account strength endurance. The results of the GS in the present investigation did not correlate to any of the other performed tests. Notwithstanding the aforementioned associations, our results indicate GS is not suitable for strength endurance evaluation of the upper body.

Another aspect which has emerged in this study, which was also highlighted in our previous investigation, is that no association is present between strength endurance and anthropometric parameters. These results are in line with other investigations $[14,35,36]$. While our BMI data did not influence the results of the tests and no significant difference was noted across the tests for RPE, a positive relation is present between BMI and RPE. These results highlight that people with a greater BMI who are required to move against a greater resistance, since the required task implies performing body weight exercises, will as a consequence have greater RPE. Such aspect has been also noted in the study of Dawes et al. [37] in which BMI was identified to influence perceptual and physiological demands of the participants and in the study of Sehl et al. [38] which noted higher RPE values in obese compared to non-obese cyclist after exercise.

It must be noted that almost no investigation previously published has adopted the parallel dips as a test for upper body strength endurance evaluation. However, the results of the present investigation demonstrate a good association with other common exercises. The PD could be adopted for a general estimate of upper body strength evaluation and therefore lead to a significant reduction of time in physical assessment. Knowledge regarding the relation between repetition cadence and performance results could be useful for a more consistent and accurate evaluation [39].

Despite the aspects discussed, this study is not without limitations. Our sample size $(n=38)$ and sample 
population (healthy young male) cannot allow us to extend the conclusions retrieved to a broader population. It is unclear if these tests could also be performed in sedentary individuals. Furthermore, it would be necessary to include objective variables, i.e. accelerometry, to confirm the associations with velocity and performance.

\section{Conclusions}

The results of the present study indicate that grip strength is not suitable to evaluate strength endurance of the upper body, while all the exercises included may be adopted to evaluate upper body strength endurance in healthy young male. However, the parallel bar dips seem to be an interesting alternative to commonly adopted tests. This test was also the only included one to possess a relation with repetition cadence. These results can be useful to sport professionals and coaches in order to simplify the assessment of strength endurance of the upper body.

\section{Acknowledgments}

The authors have no acknowledgments.

\section{Author contributions}

\section{CONCEPTION: Ewan Thomas} PERFORMANCE OF WORK: Vincenzo Gennaro INTERPRETATION OR ANALYSIS OF DATA: Marianna Bellafiore PREPARATION OF THE MANUSCRIPT: Ewan Thomas and Giuseppe Battaglia REVISION FOR IMPORTANT INTELLECTUAL CONTENT: Antonio Palma and Angelo Iovane SUPERVISION: Antonino Bianco

\section{Ethical considerations}

Each has provided informed written consent. The principles of the Italian data protection (196/2003) were guaranteed. The study was undertaken in accordance with the guidelines of the Helsinki Declaration (Hong Kong revision, September 1989) and the European Union recommendations for Good Clinical Practice (document 111/3976/88, July 1990). The study was approved by the departmental review board (C.C.S. minutes $n^{\circ} 584-2020$ approval date 24.06.2020).

\section{Conflict of interest}

The authors declare that they have no conflict of interest relevant to the content of this study.

\section{Funding}

The authors did not receive any specific grant for this research from any funding agency in the public, commercial, or not-for-profit sectors.

\section{References}

[1] Maestroni L, Read P, Bishop C, Papadopoulos K, Suchome $\mathrm{TJ}$, et al. The benefits of strength training on musculoskeleta system health: practical applications for interdisciplinary care. Sports Med. 2020. doi: 10.1007/s40279-020-01309-5k

[2] Kraschnewski JL, Sciamanna CN, Poger JM, Rovniak LS Lehman EB, et al. Is strength training associated with mortality benefits? A 15 year cohort study of US older adults. Prev Med. 2016; 87: 121-7. doi: 10.1016/j.ypmed.2016.02.038.

[3] Campbell M, Varley-Campbell J, Fulford J, Taylor B, Mileva $\mathrm{KN}$, et al. Effect of immobilisation on neuromuscular function in vivo in humans: a systematic review. Sports Med. 2019, 49(6): 931-50. doi: 10.1007/s40279-019-01088-8.

[4] Trezise J, Blazevich AJ. Anatomical and neuromuscular deter minants of strength change in previously untrained men following heavy strength training. Frontiers in Physiology. 2019 10(1001). doi: 10.3389/fphys.2019.01001.

[5] Bemben MG. Age-related alterations in muscular endurance Sports Med. 1998; 25(4): 259-69. doi: 10.2165/00007256 199825040-00004.

[6] Medical Dictionary for the Health Professions and Nursing https://medical-dictionary.thefreedictionary.com/muscular+ endurance: Retrieved June 24 2020; 2012. Muscular En durance.

[7] de la Motte SJ, Gribbin TC, Lisman P, Murphy K, Deuster PA Systematic review of the association between physical fitnes and musculoskeletal injury risk: part 2-muscular endurance and muscular strength. J Strength Cond Res. 2017; 31(11) 3218-34. doi: 10.1519/jsc.0000000000002174.

[8] Justice JN, Mani D, Pierpoint LA, Enoka RM. Fatigability o the dorsiflexors and associations among multiple domains of motor function in young and old adults. Exp Gerontol. 2014 55: 92-101. doi: 10.1016/j.exger.2014.03.018.

[9] Stafford DE, Petrofsky JS. Interactions between fatiguing and nonfatiguing isometric contractions. J Appl Physio Respir Environ Exerc Physiol. 1981; 51(2): 399-404. doi 10.1152/jappl.1981.51.2.399.

[10] Thomas E, Bianco A, Bellafiore M, Battaglia G, Paoli A, e al. Determination of a strength index for upper body local endurance strength in sedentary individuals: a cross sectional analysis. SpringerPlus. 2015; 4: 734.

[11] Bianco A, Lupo C, Alesi M, Spina S, Raccuglia M, et al. The sit up test to exhaustion as a test for muscular endurance evaluation. Springerplus. 2015; 4: 309. doi: 10.1186/s40064 015-1023-6. 
[12] Gabbett TJ, Johns J, Riemann M. Performance changes following training in junior rugby league players. J Strength Cond Res. 2008; 22(3): 910-7. doi: 10.1519/JSC.0b013e31816a5fa5.

13] Lockie RG, Ruvalcaba TR, Stierli M, Dulla JM, Dawes JJ, et al. Waist circumference and waist-to-hip ratio in law enforcement agency recruits: relationship to performance in physical fitness tests. J Strength Cond Res. 2020; 34(6): 1666-75. doi: 10.1519/jsc.0000000000002825.

[14] Thomas E, Bianco A, Raia T, Messina G, Tabacchi G, et al. Relationship between velocity and muscular endurance of the upper body. Hum Mov Sci. 2018; 60: 175-82. doi: 10.1016/j.humov.2018.06.008.

15] Kohiruimaki R, Maeo S, Kanehisa H. Suspended push-up training augments size of not only upper limb but also abdominal muscles. Int J Sports Med. 2019; 40(12): 789-95. doi: 10.1055/a-0989-2482.

16] Fielitz L, Coelho J, Horne T, Brechue W. Inter-rater reliability and intra-rater reliability of assessing the 2-minute push-up test. Mil Med. 2016; 181(2): 167-72. doi: 10.7205/MILMEDD-14-00533.

[17] Wang R, Hoffman JR, Sadres E, Bartolomei S, Muddle TW et al. Evaluating upper-body strength and power from a single test: the ballistic push-up. J Strength Cond Res. 2017. doi: 10.1519/JSC.0000000000001832.

[18] Beckham GK, Olmeda JJ, Flores AJ, Echeverry JA, Campos $\mathrm{AF}$, et al. Relationship between maximum pull-up repetitions and first repetition mean concentric velocity. J Strength Cond Res. 2018; 32(7): 1831-7. doi: 10.1519/jsc.000000000000 2431.

19] Muñoz-López M, Marchante D, Cano-Ruiz MA, Chicharro JL, Balsalobre-Fernández C. Load-, force-, and power-velocity relationships in the prone pull-up exercise. Int J Sports Physiol Perform. 2017; 12(9): 1249-55. doi: 10.1123/ijspp.2016-0657.

[20] Carlsson T, Wedholm L, Nilsson J, Carlsson M. The effects of strength training versus ski-ergometer training on doublepoling capacity of elite junior cross-country skiers. European Journal of Applied Physiology. 2017; 117(8): 1523-32. doi: 10.1007/s00421-017-3621-1.

[21] Carek PJ, Hawkins A. Rupture of pectoralis major during parallel bar dips: case report and review. Med Sci Sports Exerc. 1998; 30(3): 335-8. doi: 10.1097/00005768-19980300000001.

22] Collins SM, Silberlicht M, Perzinski C, Smith SP, Davidson PW. The relationship between body composition and preseason performance tests of collegiate male lacrosse players. J Strength Cond Res. 2014; 28(9): 2673-9. doi: 10.1519/jsc. 0000000000000454.

[23] Paoli A, Grimaldi K, D’ Agostino D, Cenci L, Moro T, et al. Ketogenic diet does not affect strength performance in elite artistic gymnasts. Journal of the International Society of Sports Nutrition. 2012; 9(1): 34. doi: 10.1186/1550-2783-9-34.

[24] Borg GA. Psychophysical bases of perceived exertion. Med Sci Sports Exerc. 1982; 14(5): 377-81.

[25] Delavier F. Strength Training Anatomy: Human Kinetics; 2010.
[26] Gottschall JS, Hastings B, Becker Z. Muscle activity patterns do not differ between push-up and bench press exercises. J Appl Biomech. 2018: 1-6. doi: 10.1123/jab.2017-0063.

[27] Snarr RL, Hallmark AV, Casey JC, Esco MR. Electromyographical comparison of a traditional, suspension device, anc towel pull-up. Journal of Human Kinetics. 2017; 58: 5-13. doi 10.1515/hukin-2017-0068.

[28] Youdas JW, Amundson CL, Cicero KS, Hahn JJ, Harezlak DT, et al. Surface electromyographic activation patterns and elbow joint motion during a pull-up, chin-up, or perfect-pullup ${ }^{\mathrm{TM}}$ rotational exercise. J Strength Cond Res. 2010; 24(12): 3404 14. doi: 10.1519/JSC.0b013e3181f1598c.

[29] Borreani S, Calatayud J, Colado JC, Moya-Nájera D, Triplet NT, et al. Muscle activation during push-ups performed unde stable and unstable conditions. Journal of Exercise Science and Fitness. 2015; 13(2): 94-8. doi: 10.1016/j.jesf.2015.07.002.

[30] Johnson D, Lynch J, Nash K, Cygan J, Mayhew JL. Relationship of lat-pull repetitions and pull-ups to maximal lat-pull and pull-up strength in men and women. J Strength Cond Res. 2009; 23(3): 1022-8. doi: 10.1519/JSC.0b013e3181a2d7f5.

[31] Zalleg D, Ben Dhahbi A, Dhahbi W, Sellami M, Padulo J, et al. Explosive Push-ups: From Popular Simple Exercises to Valid Tests for Upper-Body Power. J Strength Cond Res. 2018 doi: 10.1519/jsc.0000000000002774.

[32] Sreckovic S, Cuk I, Djuric S, Nedeljkovic A, Mirkov D, et al Evaluation of force-velocity and power-velocity relationship of arm muscles. Eur J Appl Physiol. 2015; 115(8): 1779-87. doi: 10.1007/s00421-015-3165-1.

[33] Bohannon RW. Grip strength: an indispensable biomarker for older adults. Clinical Interventions in Aging. 2019; 14: 168191. doi: 10.2147/CIA.S194543.

[34] Wind AE, Takken T, Helders PJ, Engelbert RH. Is grip strength a predictor for total muscle strength in healthy children, adolescents, and young adults? Eur J Pediatr. 2010; 169(3): 281-7. doi: 10.1007/s00431-009-1010-4.

[35] Laffaye G, Collin JM, Levernier G, Padulo J. Upper-limb power test in rock-climbing. Int J Sports Med. 2014; 35(8) 670-5. doi: 10.1055/s-0033-1358473.

[36] Vaara JP, Kyrolainen H, Niemi J, Ohrankammen O, Hakki nen A, et al. Associations of maximal strength and muscular endurance test scores with cardiorespiratory fitness and body composition. J Strength Cond Res. 2012; 26(8): 2078-86. doi 10.1519/JSC.0b013e31823b06ff.

[37] Dawes JJ, Kornhauser CL, Crespo D, Elder CL, Lindsay KG et al. Does body mass index influence the physiological and perceptual demands associated with defensive tactics training in state patrol officers? Int J Exerc Sci. 2018; 11(6): 319-30.

[38] Sehl PL, Leites GT, Martins JB, Meyer F. Responses of obese and non-obese boys cycling in the heat. Int J Sports Med. 2012, 33(6): 497-501. doi: 10.1055/s-0031-1301314.

[39] LaChance PF, Hortobagyi T. Influence of cadence on muscular performance during push-up and pull-up exercise. The Journa of Strength \& Conditioning Research. 1994; 8(2). 